\title{
IdeAs
}

Idées d'Amériques

16 | 2020

Les marges créatrices : intellectuel.le.s afrodescendant.e.s et indigènes aux Amériques, XIX-XXe siècle

\section{Los márgenes creativos: intelectuales afrodescendientes e indígenas en las Américas de los siglos XIX y XX}

Silvia Capanema, Véronique Hébrard, Fatma Ramdani y Claire Parfait

Traductor: Marta Gómez

\section{(2) OpenEdition}

\section{Journals}

Edición electrónica

URL: http://journals.openedition.org/ideas/9942

DOI: 10.4000/ideas.9942

ISSN: 1950-5701

Editor

Institut des Amériques

Referencia electrónica

Silvia Capanema, Véronique Hébrard, Fatma Ramdani y Claire Parfait, « Los márgenes creativos:

intelectuales afrodescendientes e indígenas en las Américas de los siglos XIX y XX », IdeAs [En línea],

16 | 2020, Publicado el 01 octubre 2020, consultado el 21 diciembre 2020. URL : http://

journals.openedition.org/ideas/9942; DOI : https://doi.org/10.4000/ideas.9942

Este documento fue generado automáticamente el 21 diciembre 2020.

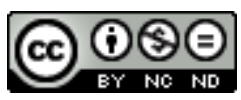

IdeAs - Idées d'Amériques est mis à disposition selon les termes de la licence Creative Commons Attribution - Pas d'Utilisation Commerciale - Pas de Modification 4.0 International. 


\title{
Los márgenes creativos:
} intelectuales afrodescendientes e indígenas en las Américas de los siglos XIX y XX

\author{
Silvia Capanema, Véronique Hébrard, Fatma Ramdani y Claire Parfait \\ Tradución : Marta Gómez
}

El presente número de la revista IdeAs nació a raíz de un taller organizado durante el Congreso del Instituto de las Américas de 2019 que llevaba por título «Los márgenes creativos: emergencia de intelectuales negros/as y mestizos/as en las Américas de los siglos XIX y XX». El taller en cuestión se organizó como continuación de un primer trabajo sobre el poder creador en los márgenes, llevado a cabo en el marco de una convocatoria de proyectos de Sorbonne Paris Cité y titulado «Escribir la historia desde los márgenes: el caso de los afroamericanos $»^{1}$. Presentado por tres profesoras investigadoras - de la Universidad Sorbonne Paris Nord (Claire Parfait), la Universidad de Paris (Marie-Jeanne Rossignol) y la Universidad Sorbonne Nouvelle-Paris 3 (Hélène Le Dantec-Lowry)-, el proyecto había evidenciado que los historiadores afroamericanos del siglo xIx y la primera mitad del siglo xx, situados en los márgenes de la sociedad, de las instituciones del saber y de las redes de edición de publicaciones, desplegaron verdaderos tesoros de la imaginación y el ingenio: ya fuera en la búsqueda de fuentes o adoptando fórmulas alternativas para publicar y dar a conocer sus trabajos, estos historiadores fueron capaces de innovar y de ser creativos desde su posición marginal.

2 Este número de IdeAs amplía la cuestión a otras áreas geográficas y culturales y a otros márgenes al objeto de examinar las Américas en su conjunto -América Latina, el Caribe y América del Norte- y explorar la riqueza potencial de un enfoque comparativo. El dossier atiende a protagonistas negros, «mestizos» o indígenas, según la terminología de los países o de las distintas áreas estudiadas ${ }^{2}$, e incorpora la perspectiva de género. Además, si bien engloba diversos trabajos centrados en historiadores e historiadoras, el 
número de la revista aborda de manera más general los procesos de creación y difusión desde los márgenes. En este sentido, el término «intelectual» debe interpretarse aquí en su acepción más amplia, referida a los estudiosos (entre ellos, los historiadores), los artistas y los escritores ${ }^{3}$, pero también a autodidactas y militantes. Por tanto, no son necesariamente expertos, sino más bien hombres y mujeres de cultura, creadores y mediadores ${ }^{4}$, parafraseando la definición dada por Pascal Ory y Jean-François Sirinelli. Este número no pretende ser exhaustivo: ofrece una serie de estudios de caso $\mathrm{y}$, en conjunto, algunos países son más estudiados que otros, como es el caso de Brasil. El objetivo es ofrecer pistas para explorar la cuestión de la fuerza creadora en los márgenes. Al igual que el propio concepto de «intelectual», cuya definición es objeto de debate, también debemos precisar la idea misma de «margen». La marginalidad puede ser, de hecho, de género, racial, étnica, social, espacial, económica, profesional, política, cultural o académica. Puede considerarse negativa, sinónimo de exclusión y relegación, pero también puede percibirse como lugar para la creación. Así lo ve de hecho Michel Wieviorka:

Desde hace un tiempo, las ciencias sociales vienen aplicando enfoques ambivalentes o contradictorios en relación con los márgenes y la marginalidad. Los más clásicos ven en ello una serie de problemas y dificultades que afectan a determinadas personas o grupos o que constituyen una amenaza para la sociedad, y examinan el origen, la producción y las fuentes de esos problemas y dificultades. Otros enfoques, en cambio, adoptan otra mirada frente a los márgenes y la marginalidad y plantean, casi al contrario, su contribución a la sociedad, e incluso al mundo, y a sus normas, así como a su creatividad, su vitalidad y su dinamismo ${ }^{5}$.

3 La experiencia de América del Norte, Estados Unidos y Canadá basada en la aportación de historiadores/as negros/as representa el primer nivel de un enfoque que combina marginalidad y originalidad o creación como una manera de sortear los márgenes, pero también de responder de otro modo a las cuestiones de la sociedad desde otro espacio de experiencia. Sin embargo, hablar de márgenes nos lleva a reflexionar sobre la existencia de condiciones que pueden ser doblemente, e incluso triplemente, marginales: pertenecer a una sociedad marginal en el orden mundial occidental (América Latina, por ejemplo) y situarse en la periferia dentro de esa sociedad por razones de raza, género o condición social.

El desplazamiento de la mirada desde el centro a los márgenes es una cuestión central de los debates de los estudios poscoloniales desarrollados sobre todo en el mundo anglosajón ${ }^{6}$, pero también de las corrientes de pensamiento decolonial surgidas en su mayoría en América Latina y Canadá. La emergencia de estos intelectuales de los márgenes en distintos espacios y épocas del continente americano permite comparar las distintas perspectivas, más o menos presentes en los diferentes artículos del dossier. La articulación entre género, raza y clase se encuentra, así pues, en el centro de los análisis. La creación intelectual -ya sea literaria, científica o artística- es el elemento que permite tal descentralización, así como producir lo nuevo y escribir la historia de los olvidados/as o, como mínimo, la historia y el relato alimentados por experiencias vividas en los márgenes o por vivencias marginales en sí mismas. De ahí la plasticidad de la noción de «margen», que no es solo geográfica, anclada en un territorio y delimitada por fronteras, sino también simbólica.

5 Los intelectuales de los márgenes son verdaderos «pasantes culturales» que llevan a cabo esta transfiguración de los sentidos, a veces como experiencias concretas de 
fenómenos de «creolización» ${ }^{7}$, descritos, por ejemplo, en el artículo de Maud Delevaux a propósito del poeta afroperuano Nicomedes Santa Cruz.

6 Los amerindios, cuando escriben su historia y se convierten deliberadamente en «intelectuales indígenas», no solo producen excelentes estudios, sino que son también portadores de una nueva epistemología, tal y como muestran el texto de Stéphanie Boutevin sobre la visión autóctona del mundo en Canadá y el análisis de Lionel Larré sobre la reconquista de la soberanía intelectual por parte de los indígenas de Estados Unidos. Los intelectuales indígenas son iniciadores de proyectos innovadores en materia de prácticas culturales y de educación, como muestra el artículo de Elena Nava Morales sobre la creación de la comunalidad, una experiencia desarrollada por intelectuales indígenas del Estado de Oaxaca (México) en la década de 1980 y retomada por otros actores contemporáneos de los ámbitos universitario y cultural. El propio «intelectual indígena» Luiz Henrique Eloy Amado explora los profundos cambios que se producen dentro de las ciencias humanas cuando los indígenas se matriculan en las universidades y se lanzan a investigar sobre el terreno. Los indígenas ya no son solo «objeto de investigación», sino que se convierten también en productores de conocimiento científico, modificando además la forma de pensar de sus contemporáneos. Algo similar se produce con los intelectuales negros, objeto del artículo de Claire Parfait, que traza la historia -durante mucho tiempo «olvidada»- de Joel Augustus Roger, historiador negro norteamericano de la primera mitad del siglo xx que se inscribe $-\mathrm{y}$ al mismo tiempo se desmarca- en una larga tradición de historiadores negros. Por su parte, Fatma Ramdani estudia a la historiadora afroamericana Mary Church Terrell quien, a finales del siglo XIX y principios del xx, redactó ensayos y una autobiografía con el fin de reivindicar un espacio para el testimonio de las mujeres negras en la narrativa de una América segregada.

7 Las mujeres, posicionadas a veces en una doble o triple marginalidad (mujeres, racializadas y pobres) son agentes de transgresión literaria o política. Producen una nueva estética, pero también dan muestras de un compromiso transformador, como muestra el artículo de Grégory Bekhtari sobre la acción política y sindical de las mujeres negras, trabajadoras domésticas, en los Estados Unidos de mediados del siglo $\mathrm{xx}$, cuyas luchas son abanderadas por la corriente Black Left Feminism. Estas mujeres transforman su condición marginal en motor para la vanguardia política y cultural.

Las mujeres negras intelectuales se convierten en escritoras de su historia y en fuente principal para otra historia. Además, son las creadoras de nuevas estéticas literarias, como muestra la autora Silvia Capanema en su análisis cruzado de los textos de Carolina Maria de Jesus y Conceição Evaristo sobre sus vivencias en las favelas brasileñas del siglo xx. Esta relación entre nueva estética literaria y fuente para la historia aparece también en los escritos de Estercilia Simanca Pushaina, revelando las estrategias de negociación de la comunidad indígena wayúu, instalada en la península de La Guajira, entre Colombia y Venezuela. Esto es lo que desvela el artículo de Laura Lema Silva. La elección del idioma dominante, el español, obedece a un deseo deliberado de diálogo con la sociedad colombiana, así como de transmisión.

Del mismo modo, las mujeres indígenas intelectuales utilizan la escritura para expresar su compromiso y transmitir sus tradiciones, como muestra el ensayo de Fabrice Le Corguillé sobre el caso de Sarah Winnemucca en los Estados Unidos del siglo XIX.

10 La autora Nádia Maria Cardoso da Silva analiza las estrategias de negociación y afirmación de dos intelectuales afrobrasileños invisibilizados: Virginia Leone Bicudo y 
Guerreiros Ramos. Gracias a su situación marginal, ambos son precursores de un pensamiento decolonial. De igual forma, el escritor Cyril Lionel Robert James rompe durante su juventud caribeña con la lógica colonialista y, a partir de la década de 1920, propone una lectura para "provincializar Europa», utilizando la expresión formulada en 2000 por el historiador Dipesh Chakrabarty ${ }^{8}$.

11 Valmir Luis Saldanha da Silva analiza a través de un largo periodo de tiempo la creación literaria de los afrodescendientes, desde escritores consagrados como Machado de Assis y Gonçalves Dias hasta el grupo de rap Racionais Mc's. El autor del artículo demuestra que es precisamente en la descentralización y la transformación de la periferia en el centro donde reside la principal contribución del poder creador de los márgenes. Este desplazamiento de los sujetos periféricos al centro es el tema principal del trabajo del intelectual universitario africano instalado en Brasil Kabengele Munanga (artículo de Reinaldo José de Oliveira y Regina Marques de Souza Oliveira).

Por último, la descentralización de los lugares y la mirada se convierte en un elemento que permite a cualquier intelectual reexaminar el mundo: eso es lo que revela el análisis que, a modo de colofón, realiza Françoise Palleau-Papin a propósito de la escritura del escritor norteamericano Ben Fountain cuando utiliza a Haití como espejo de los Estados Unidos.

Todas estas experiencias de creación desde los márgenes, materializadas de múltiples maneras en el mundo americano, tienen cabida en este dossier temático. Los intelectuales citados en los artículos son a un tiempo observadores y actores, siempre comprometidos y, en ese sentido, el dossier insta a reflexionar sobre la relación entre marginalidad y militancia y, en otro orden de cosas, invita a analizar la pertinencia de los conceptos de centro y periferia. En efecto, ¿puede el concepto de «margen» seguir teniendo sentido ahora que las nociones de historia cruzada o conectada y de circulación han sustituido a las de historia de la nación y de las transferencias, haciendo que la oposición entre «centro» y «periferia» sea sin duda menos pertinente?

\section{NOTAS}

1. Véase https://hdlm.hypotheses.org/ y las publicaciones asociadas: Writing History from the Margins: African Americans and the Quest for Freedom, bajo la dirección de Claire Parfait, Hélène Le Dantec-Lowry y Claire Bourhis-Mariotti (New York: Routledge, 2017); Écrire l'histoire depuis les marges : une anthologie d'historiens africains-américains 1855-1865, colección SHS, Terra HN éditions, 2018: http://www.shs.terra-hn-editions.org/Collection/?-Historiens-africains-americains; Histoire en marges. Les périphéries de l'histoire globale, editado por Hélène Le Dantec-Lowry, Matthieu Renault, Marie-Jeanne Rossignol y Pauline Vermeren, Tours, Presses universitaires FrançoisRabelais, 2018.

2. Retomando las palabras de Pap Ndiaye, «Las razas no existen por sí mismas, sino como categorías imaginarias construidas históricamente (...). La noción de «mestizo» no tiene más fundamento científico que la noción de raza, ya que presupone la existencia previa de razas puras. Por tanto, se la considera en un sentido histórico y social», Pap N ‘Diaye, «Controverse, Pour une histoire des populations noires en France : préalables théoriques ", Le Mouvement Social, 
no 213, octubre-diciembre de 2005: 91-108; sobre este tema, véase también: Magali Bessone, Sans distinction de race? Une analyse critique du concept de race et de ses effets pratiques, Paris, Vrin, " Philosophie concrète ", 2013.

3. Definición de «intelectuales» dada por Vincent Duclert, quien aun así señala que dicha definición es «limitativa»: Vincent Duclert, «Les intellectuels, un problème pour l'histoire culturelle», Les Cahiers du Centre de Recherches Historiques [En línea], 31 | 2003, publicada en línea el 15 de septiembre de 2008, consultado el 2 de septiembre de 2020. URL: http:// journals.openedition.org/ccrh/293; DOI: https://doi.org/10.4000/ccrh.293

Cabe recordar por otra parte que la definición del término «intelectual» varía según el lugar y la época, tal y como señala François Dosse: «Puede por tanto afirmarse que la noción de «intelectual» es polisémica y abarca concepciones diferentes según los periodos y las áreas civilizacionales», François Dosse, «Introduction», La marche des idées: Histoire des intellectuels, histoire intellectuelle, París, La Découverte, 2012.

4. Pascal Ory y Jean-François Sirinelli, Les intellectuels en France. De l'affaire Dreyfus à nos jours, París, Armand Colin, 1986, p. 5. Estos autores añaden que este «hombre de cultura se presenta como hombre político, creador o consumidor de ideología» (Ibid.).

5. Michel Wieviorka, «Ce que sont les marges aux sciences sociales», Conferencia inaugural del coloquio internacional «El reconocimiento de los márgenes en el centro de las dinámicas sociales en Francia y Japón», 1 de julio de 2016 - Casa franco-japonesa de Tokio: https:// wieviorka.hypotheses.org/706

6. Véase: Achille Mbembe, «Qu'est-ce que la pensée postcoloniale ? (Entrevista)», Esprit [En línea], marzo de 2019, consultado el 27 de septiembre de 2020. Véase también: Edward W. Said, Orientalism, New York, Pantheon Books, c. 1978; Paul Gilroy, The Black Atlantic: Modernity and Double Consciousness, London, Verso, 1993.

7. Édouard Glissant, Introduction à une poétique du Divers [Introducción a una poética de lo diverso], París, Gallimard, 1996.

8. Chakrabarty, Dipesh. Provincializing Europe: Postcolonial Thought and Historical Difference. Princeton, Oxford, Princeton University Press, 2000.

\section{AUTORES}

\section{SILVIA CAPANEMA}

Docteure en Histoire par l'EHESS, Paris, Maîtresse de conférences à l'Université Paris 13 -

Sorbonne Paris Nord

\section{VÉRONIQUE HÉBRARD}

Véronique Hébrard est Professeure d'histoire et civilisation de l'Amérique latine contemporaine à l'Université de Lille. Ses travaux portent sur l'histoire politique du Venezuela au XIX ${ }^{\mathrm{e}}$ siècle et plus spécifiquement sur les processus de mobilisation et de politisation des populations civiles en temps de guerre. Sur ces questions elle a notamment publié «Ciudades leales, ciudades patriotas. Guerra de independencia y identidades urbanas (Venezuela, siglo XIX) », Tiempo y Espacio (Caracas), n 63, enero-junio 2015, p. 163-186; 21; « Justicia “excepcional” y lógicas de radicalización durante la primera Pacificación de Venezuela (1812-1814) », Revista de Indias, 2016, vol. LXXVI, no 266, p. 17-50.Elle s'intéresse également aux archives des américanistes français, sur 
lesquelles elle a publié : Véronique Hébrard (dir.), Por una concepción atlántica del americanismo. En los pasos de François Chevalier, Paris, Editions des Archives Contemporaines, 2013 ; Véronique Hébrard (dir.), Sur les traces d'un mexicaniste français. Constitution et analyse du fonds François Chevalier, Paris, Karthala, collection Pollens, 2005.

\section{FATMA RAMDANI}

Fatma Ramdani est maîtresse de conférences à l'Université de Lille en histoire des États-Unis. Ses travaux portent sur les mouvements féministes américains dans leur dimension internationale. Elle s'intéresse à la contribution des Africaines-Américaines dans l'écriture de l'histoire. Ses travaux s'interrogent sur les questions de transmission et de mémoire. A titre d'exemple, a publié « Mary McLeod Bethune (1875-1955), historienne africaine américaine oubliée », dans Histoire en marges, Les périphéries de l'histoire globale, ed. Hélène Le Dantec-Lowry et al., Presses Universitaires François Rabelais, 2018.

\section{CLAIRE PARFAIT}

Claire Parfait est affiliée à la Pléiade, Université Sorbonne Paris Nord 